\title{
Sustained JNK signaling by proteolytically processed HPK1 mediates IL-3 independent survival during monocytic differentiation
}

\author{
R Arnold ${ }^{\star, 1,2}$, CR Frey ${ }^{2}$, W Müller², D Brenner ${ }^{2}$, PH Krammer ${ }^{2}$ and F Kiefer, ${ }^{\star, 1,3}$
}

We studied monocytic differentiation of primary mouse progenitor cells to understand molecular mechanisms of differentiation. We found a tightly controlled non-apoptotic activation of caspase-3 that correlated with differentiation. Although caspase activity was already detected during monocytic differentiation, a caspase-3 target has not been identified yet. We show that hematopoietic progenitor kinase 1 (HPK1) is processed towards its $\mathrm{N}$ - and C-terminal fragments during monocytic differentiation. While HPK1 is an immunoreceptor-proximal kinase in T and B cells, its role in myeloid cells is elusive. Here, we show that the N-terminal cleavage product, HPK1-N, comprising the kinase domain, confers progenitor cell survival independent of the growth factor IL-3. Furthermore, HPK1-N causes differentiation of progenitor cells towards the monocytic lineage. In contrast to full-length kinase, HPK1-N is constitutively active causing sustained JNK activation, Bad phosphorylation and survival. Blocking of caspase activity during differentiation of primary mouse progenitor cells leads to reduced HPK1-N levels, suppressed JNK activity and attenuated monocytic differentiation. Our work explains growth factor-independent survival during monocytic differentiation by caspase-mediated processing of HPK1 towards HPK1-N.

Cell Death and Differentiation (2007) 14, 568-575. doi:10.1038/sj.cdd.4402042; published online 6 October 2006

Within the hematopoietic system lineage commitment, differentiation and proliferation are precisely balanced throughout life, resulting in adjusted levels of myeloid and lymphoid cells. A complicated network of cytokines essentially contributes to hematopoietic homeostasis. Myeloid progenitor cells and myeloid-like cell lines respond to the cytokine interleukine-3 (IL-3) with proliferation and survival. ${ }^{1}$ While removal of IL-3 results in cell death via apoptosis, suppression of apoptosis allows differentiation of the mouse hematopoietic progenitor cell line FDC-P1 in the absence of IL-3. ${ }^{2}$ Monocytic differentiation of primary bone marrow and fetal liver progenitors or myeloid cell lines can be induced by IL-3 withdrawal and stimulation with monocyte-colony stimulating factor (M-CSF). ${ }^{3}$ Monocytic differentiation is accompanied by caspase activation; ${ }^{4,5}$ however, the molecular targets of caspase activity during monocytic differentiation have not been identified.

Hematopoietic progenitor kinase 1 (HPK1) is comprised of a catalytic domain with extensive homology to the Sterile 20 kinase of the yeast Saccharomyces cerevisiae and a Cterminally located regulatory domain, called citron homology domain. ${ }^{6}$ Ectopic expression renders full-length HPK1 active in epithelial cells resulting in selective activation of the JNK and the NF $\kappa$ B pathways. ${ }^{7,8}$ Upon caspase-3 cleavage the
$\mathrm{N}$-terminal kinase domain of HPK1 (HPK1-N) is separated from the C-terminus (HPK1-C) resulting in suppression of $\mathrm{NF}_{\kappa} \mathrm{B} .{ }^{8}$ In non-stimulated lymphocytes, HPK1 kinase activity is hardly detectable, but antigen receptor crosslinking leads to profound HPK1 activation. ${ }^{9,10}$ HPK1 activation involves binding of cell-specific adaptor proteins, relocation to the plasma membrane, autophosphorylation and transphosphorylation by protein kinase D1. ${ }^{1-13}$ While a role for HPK1 in the regulation of T-cell apoptosis was already suggested, ${ }^{14}$ our recent studies have shown proteolytic processing of HPK1 into HPK1-C in non-apoptotic preactivated primary T cells. ${ }^{15}$ In this study, the cleavage product HPK1-C sensitizes towards T-cell receptor-mediated cell death, while full-length HPK1 enables activation and survival of T cells. In contrast to the studies in lymphoid cells, which suggest that HPK1 acts as a life/death switch, relatively little is known about the role of HPK1 in myeloid cells.

Here we show a tightly controlled, non-apoptotic activation of caspase-3 during monocytic differentiation of primary mouse progenitor cells that correlates with proteolytic processing of HPK1. The presence of HPK1-N results in cytokine-independent survival of FDC-P1 myeloid progenitor cells and upregulation of markers for monocytic differentiation. While kinase activity of full-length HPK1 depends on IL-3

\footnotetext{
${ }^{1}$ Max-Planck-Institute for Physiological and Clinical Research, WG Kerckhoff-Institute, Parkstrasse 1, D-61231 Bad Nauheim, Germany; ${ }^{2}$ Tumor Immunology Program, German Cancer Research Center, Im Neuenheimer Feld 280, D-69112 Heidelberg, Germany and ${ }^{3}$ Max-Planck-Institute for Molecular Biomedicine, Von-EsmarchStrasse 56, D-48149 Münster, Germany

*Corresponding authors: R Arnold, German Cancer Research Center (DKFZ), INF 280, D-69112 Heidelberg, Germany. Tel: + 496221423769 ; Fax: + 496221411 715; E-mail: r.arnold@ dkfz.de or F Kiefer, MPI for Molecular Biomedicine, Von-Esmarch-Strasse 56, D-48149 Münster, Germany. Tel: + 492518357181 ;

Fax: + 49251835 8616; E-mail: fkiefer@ gwdg.de

Keywords: caspase-3; myeloid progenitor cells; apoptosis; FDC-P1

Abbreviations: JNK, C-Jun N-terminal kinase; IL-3, interleukine-3; HPK1, hematopoietic progenitor kinase 1; M-CSF, monocyte-colony stimulating factor; RT-PCR, reverse transcriptase polymerase chain reaction; Anx V, annexin V; MPO, myeloperoxidase; z-VAD-fmk, benzoyl-valine-alanine-aspartate-fluoromethylketone; PI, propidium iodide

Received 02.3.06; revised 02.8.06; accepted 08.8.06; Edited by R De Maria; published online 06.10.06
} 
stimulation in FDC-P1 cells, HPK1-N is a constitutive active kinase causing sustained JNK activity. Thereby, apoptosis of HPK1-N bearing cells is prevented by phosphorylation of Bad and differentiation can proceed. Our work suggests HPK1 to be a molecular target of caspase-3 activity during monocytic differentiation.

\section{Results}

Activation of caspase-3 during monocytic differentiation of primary mouse progenitor cells. We induced in vitro differentiation of primary mouse progenitor cells to better understand the molecular mechanisms underlying monocytic differentiation (Figure 1a). Unfractionated mouse bone marrow or fetal liver cells were used as starting population. During a 48-h preculture period, monocytic progenitors remained in suspension and were subsequently separated from differentiated adherent cells. Remaining progenitor cells were further differentiated after removal of IL-3 for 7 days in the presence of M-CSF. During this in vitro differentiation the percentage of cells staining positive for the differentiation markers F4/80 and Mac-1 (CD11b) constantly increased (Figure 1b). By day 9 most of the cells displayed a macrophage-like morphology and were adherent to the cell culture dish (data not shown). During this period the level of apoptotic cells in our in vitro differentiation system staining positive for annexin $\mathrm{V}(\mathrm{Anx} \mathrm{V})$ remained rather constant (Figure 1b). In contrast, we detected the temporally tightly controlled appearance of the active p17/p19 fragments of caspase-3 around days 3-6 (Figure 1c), suggesting a nonapoptotic caspase activity during monocytic differentiation, which has been previously reported. ${ }^{4,5}$ Accordingly, application of the caspase inhibitor z-VAD-fmk during in vitro culture of progenitor cells blocks monocytic differentiation. ${ }^{4}$ However, a caspase-3 target involved in monocytic differentiation has not been identified yet.

HPK1 is a caspase-3 substrate during monocytic differentiation. In search of caspase-3 substrates, which are cleaved during in vitro differentiation of primary mouse progenitors, we found HPK1 to be processed into its $\mathrm{N}$ - and C-terminal fragments (Figure 1d). Accumulation of the HPK1 cleavage products at days 5 and 6 of differentiation is seen just after peaking of active caspase-3 at day 4 . Therefore, endogenous HPK1 might be a target of active caspase-3 during monocytic differentiation in vitro. In contrast, we did not find any cleavage of the apoptosis-related caspase-3 target PARP (data not shown). This result further
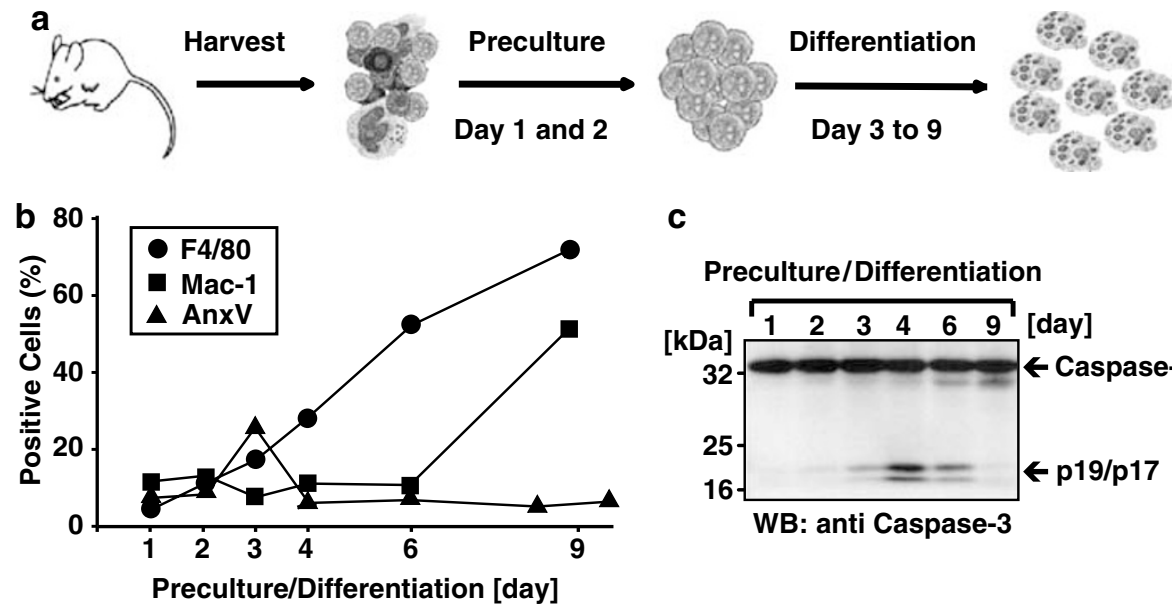

C

Preculture/Differentiation
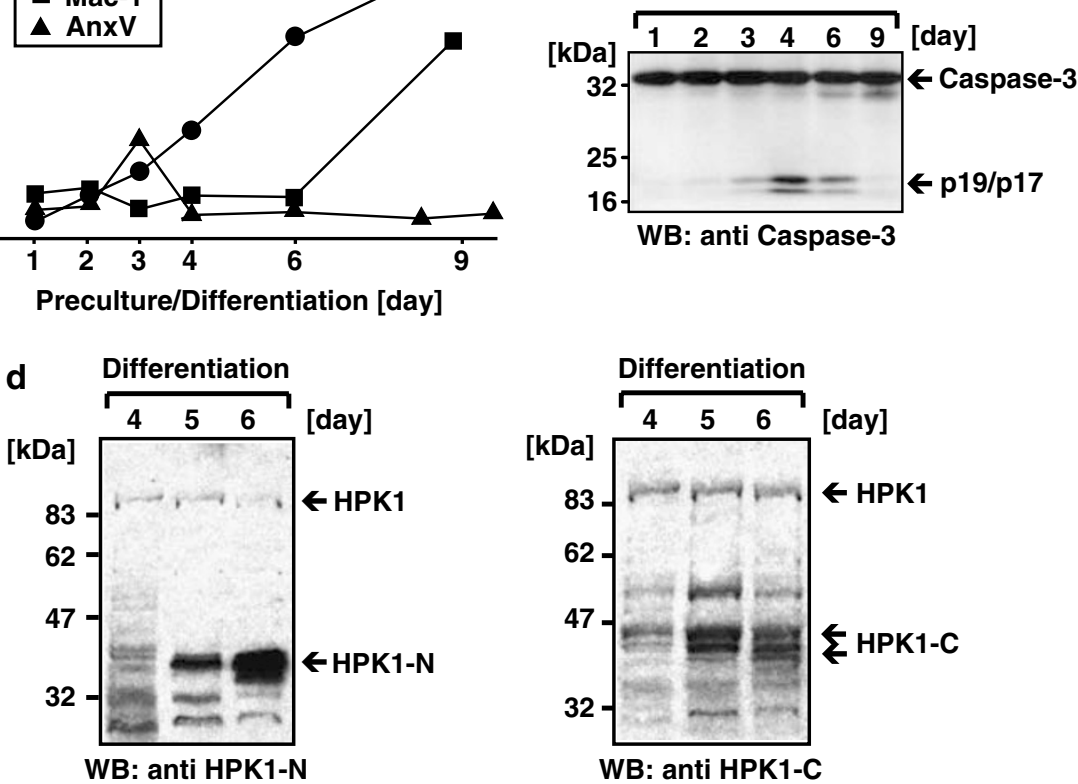

Figure 1 Activation of caspase-3 and cleavage of HPK1 correlates with monocytic differentiation of primary mouse progenitor cells. (a) Schematic overview of the in vitro differentiation regimen for primary mouse progenitor cells towards the monocytic lineage. Myeloid progenitor cells were expanded by preculture of total bone marrow or fetal liver in the presence of IL-3 and M-CSF, subsequently progenitor cells were separated from adherent (differentiated) cells which were excluded from the in vitro differentiation analysis. For monocytic differentiation cells were cultured in the presence of M-CSF for 7 days. (b) At various times during the differentiation cells staining positive for the macrophage markers F4/80 or Mac-1 (CD11b) or the apoptosis marker annexin V (Anx V) were enumerated by flow cytometry. (c and d) At the indicated times cell lysates were subjected to Western blotting (WB) with antibodies against caspase-3, HPK1-N or HPK1-C. Results are representative of three repeats 
strengthens a non-apoptotic role for caspase-3 during monocytic differentiation, which is supported by the absence of significant apoptosis during our in vitro culture (Figure 1b). Therefore, our data suggest that cleavage of HPK1 by caspase-3 is implicated in monocytic differentiation.

\section{IL-3 independent survival of progenitor cells harboring} HPK1-N. To further analyze the role of the HPK1 cleavage fragments, we took advantage of the mouse myeloid progenitor cell line FDC-P1 $1^{16}$ and using retroviral transduction generated cell clones stably expressing either HPK1-N or HPK1-C (Figure 2a). Five clones (N-1 to N-5 and $\mathrm{C}-1$ to $\mathrm{C}-5$, respectively) were pooled for subsequent analysis to avoid effects of clonal variation and compared to pooled clones containing the virus without insert (vector). While the HPK1-N expressing cell pool displayed a slightly delayed proliferation rate (Figure $2 b$ ), the presence of HPK1$\mathrm{N}$ did not influence viability of FDC-P1 cells in the presence of IL-3 (Figure 2c).

Growth and survival of the hematopoietic progenitor cell line FDC-P1 is strictly IL-3 dependent ${ }^{2}$ and apoptosis can be induced by IL-3 withdrawal. Therefore, we subjected the pooled HPK1-N and HPK1-C cell clones to IL-3 deprivation, resembling the shortage of this growth factor in the in vitro differentiation system applied (Figure 1a, days 3-9). Interestingly, the HPK1-N cell pool was largely resistant towards apoptosis-induction by IL-3 withdrawal (Figure 2c). In contrast, the HPK1-C expressing cells mildly increased the sensitivity towards cell death, which was also seen previously in lymphocytes. ${ }^{15}$ Prolonged culture of HPK1-N cells without IL-3 also significantly decreased viability (Figure $2 \mathrm{~d}$ ). This result shows that HPK1-N cells are largely apoptosisresistant, but not completely IL-3 independent, a state that would likely require additional factors. Testing this idea, we found that the addition of macrophage-colony stimulating factor (M-CSF) increased survival of HPK1-N cells. While vector transduced FDC-P1 cells did not respond to this cytokine (Figure 2d), FDC-P1 cells do not express the receptor for M-CSF (M-CSFR) and can only be rendered responsive to M-CSF by differentiation induction or by ectopic expression of the M-CSFR. ${ }^{20}$ Therefore, it was likely that the presence of HPK1-N induced the expression of M-CSFR.

HPK1-N induces differentiation towards the monocytic lineage. We reasoned that the phenotypic differences of HPK1-N cells might be due to the induction of monocytic differentiation. May-Grünwald-Giemsa staining of HPK1-N cells revealed a higher granularity as compared to vector transduced FDC-P1 cells (Figure 3a, upper panel). In addition, HPK1-N cells were bigger in cell size (Figure $3 a$, upper panel and Figure $3 \mathrm{~b}$ ) and displayed an increased tendency to adhere to the cell culture dishes during regular culture (Figure 3a, lower panel). In permanently growing cultures of HPK1-N cells, we detected a constant ratio of adherent and suspended cells. In cultures of previously only adherent cells, cells detached until the equilibrium between
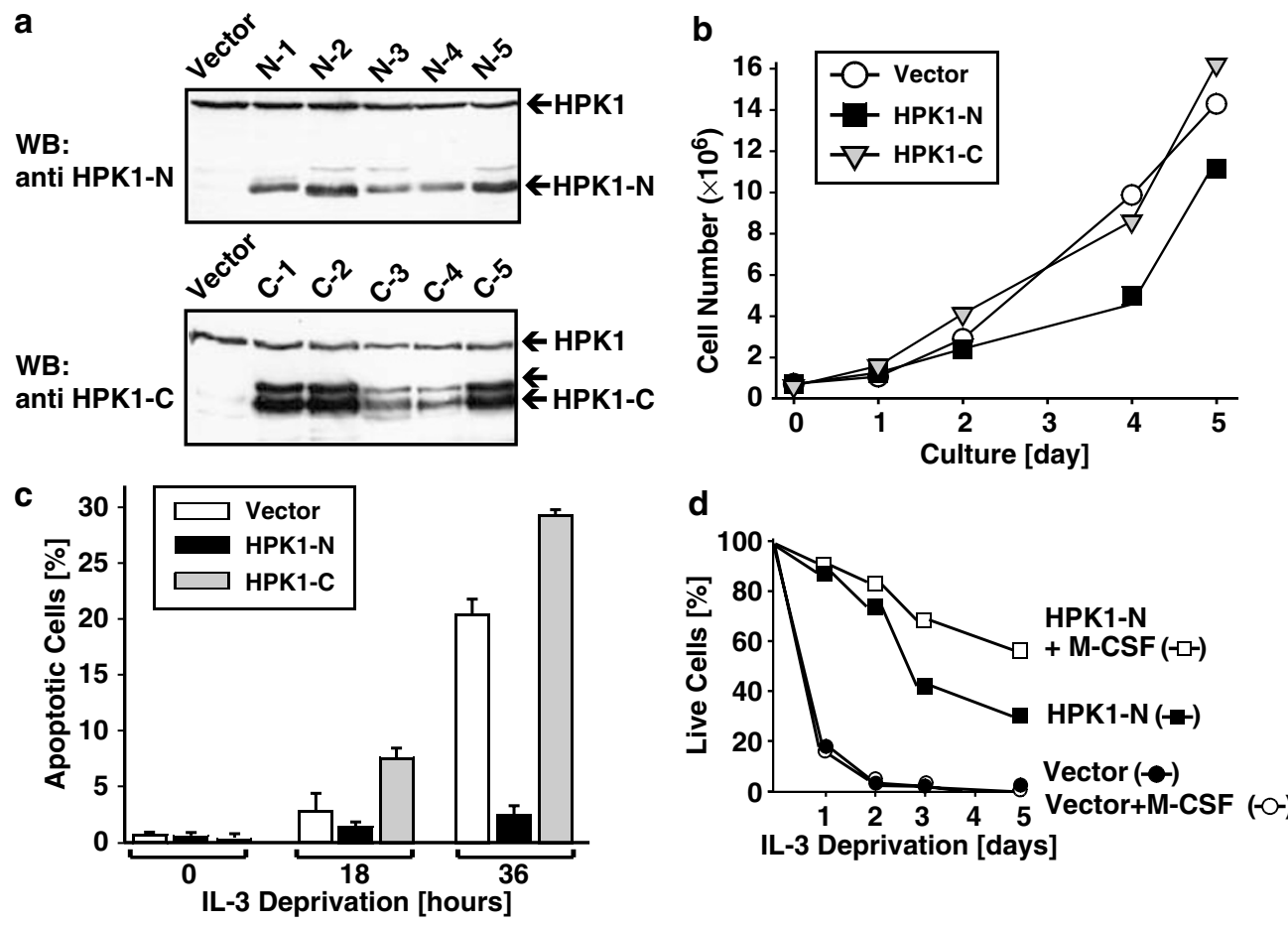

Figure 2 HPK1-N mediates IL-3 independent survival of progenitor cells. (a) Hematopoietic progenitor cell line FDC-P1 was used to generate cell clones stably transduced with either HPK1-N or HPK1-C or the neomycin resistance only (vector). Expression of endogenous HPK1 and ectopic HPK1-N or HPK1-C is shown for various FDC-P1 cell clones (N-1 to N-5 or C-1 to C-5). For subsequent analysis, the depicted clones were pooled to avoid clonal variation and subjected to cumulative cell growth analysis (b) or interleukine-3 (IL-3) deprivation (c and d) for the indicated time periods. Apoptotic cells were detected by analysis of their sub-G1 DNA content using flow cytometry. Life cells were identified by trypan blue staining. Where indicated macrophage-colony stimulating factor (M-CSF) was added. Values given depict a representative experiment of four independent repeats with identical outcome. Standard deviations were calculated from triplicate measurements 

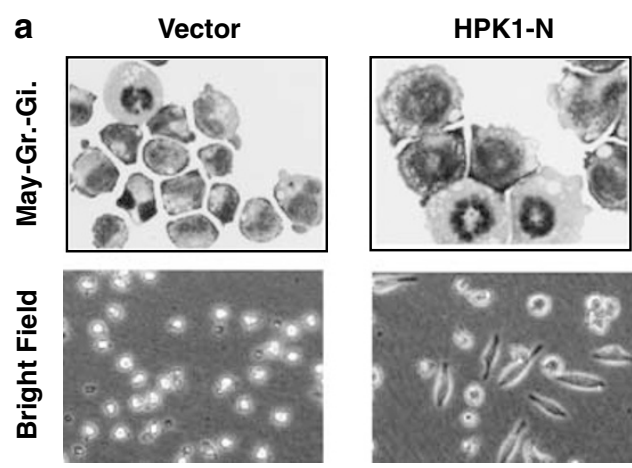

b

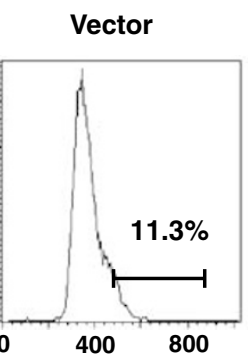

Forward Scatter

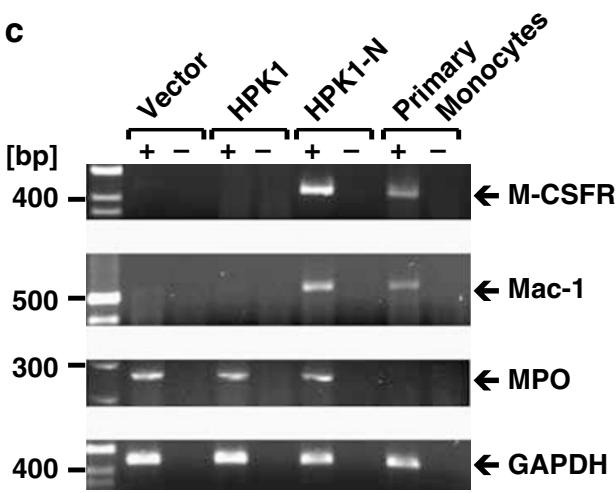

d

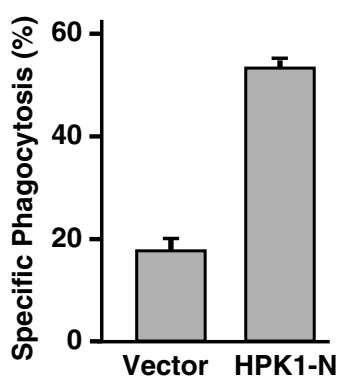

Figure 3 HPK1-N causes differentiation of progenitor cells towards the monocytic lineage. (a) HPK1-N or vector transfected FDC-P1 cell pools were analyzed by MayGünwald-Giemsa (May-Gr.-Gi.) staining to detect differences in cell granularity (upper panel). Bright field microscopy images show attachment of HPK1-N cells to the tissue culture plastic during regular culture (lower panel). (b) Cell size was analyzed by flow cytometry and compared as forward scatter histograms for HPK1-N or vector transduced FDC-P1 cell pools during regular culture. The percentage of cells within the range of the marker is indicated. (c) Expression of the monocytic markers M-CSFR or Mac-1 (CD11b) was analyzed by RT-PCR for the indicated FDC-P1 cell pools. Expression of MPO and GAPDH served as controls. Analysis was performed either with ( + ) or without $(-)$ addition of reverse transcriptase. Primary in vitro differentiated monocytes at day 9 of culture (see Figure 1) were included as control. (d) Phagocytosis was analyzed by flow cytometry after incubation of HPK1-N or vector control cells with fluorescent beads. Standard deviation was calculated from triplicate measurements

adherent and suspended cells was reestablished. Similarly after reseeding of suspension cells, cells attached to reestablish the equilibrium. Compared to previous reports of in vitro differentiated FDC-P1 cells, ${ }^{20-22}$ the observed morphological changes indicate monocytic differentiation of HPK1-N cells.

To address the question, whether the adherence of HPK1$\mathrm{N}$ cells might influence their susceptibility towards apoptosis, suspended cells were collected and adherent cells were released from the Petridish by pipetting, deprived of IL3 and reseeded. After $18 \mathrm{~h}$ the rate of apoptosis was significantly higher in cells previously growing in suspension (data not shown). This again suggests that cytokine-independent survival of HPK1-N cells is linked to the adherence-phenotype and thereby to ongoing differentiation of these cells.

To examine the presence of the monocytic differentiation markers M-CSFR or Mac-1 (CD11b) in HPK1-N cells, we used RT-PCR. Addition of M-CSF rescued HPK1-N cells from apoptosis after IL-3 withdrawal (Figure 2d) and we detected expression of M-CSFR message in HPK1-N cells at similar levels as in in vitro generated primary monocytes (Figure $3 \mathrm{c}$ ). In addition, HPK1-N cells were also positive for the differentiation marker Mac-1. FDC-P1 cells containing empty vector or elevated expression of $\mathrm{HPK} 1^{8}$ did not show Mac-1 expression (Figure $3 \mathrm{c}$ ). Furthermore, HPK1-N cells stained positive for the differentiation marker F4/80 (data not shown). These results further support our hypothesis that HPK1-N drives FDC-P1 cells towards monocytic differentiation.
Finally, we tested for functional monocytic differentiation and found HPK1-N cells capable of enhanced phagocytosis compared to vector control cells (Figure $3 \mathrm{~d}$ ). This result shows that HPK1-N cells have acquired functional properties typical of the monocytic lineage and suggests a biological role for HPK1-N in mediating monocytic differentiation of progenitor cells.

Antiapoptotic Bad phosphorylation and sustained JNK activation by HPK1-N. To understand the role of HPK1-N during myeloid differentiation on the molecular level, we next analyzed affected signaling pathways. Activation of the JNK pathway is known to mediate IL-3 dependent survival of the hematopoietic progenitor cell line FDC-P1. ${ }^{23}$ In addition, JNK activity has been reported to be necessary for myeloid differentiation. ${ }^{24}$ Therefore, we first analyzed the kinase activity of endogenous JNK proteins in HPK1-N cells compared to stable FDC-P1 cell pools containing HPK1-C or empty vector (Figure 4a). We found that already under normal growth conditions HPK1-N cells showed a robust four-fold elevation of JNK activity, while in HPK1-C cells JNK activity was only slightly enhanced. This observation was also made in previous studies showing robust JNK activation by ectopic expression of the liberated kinase domain of HPK1 in epithelial cells. ${ }^{8,25}$ Furthermore, this result suggests that survival and differentiation of HPK1-N cells might be linked to activation of the JNK pathway. 
a

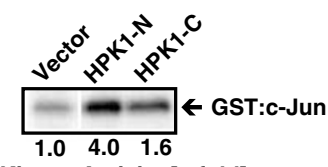

JNK Kinase Activity [x-fold]

WB: anti JNK1/2

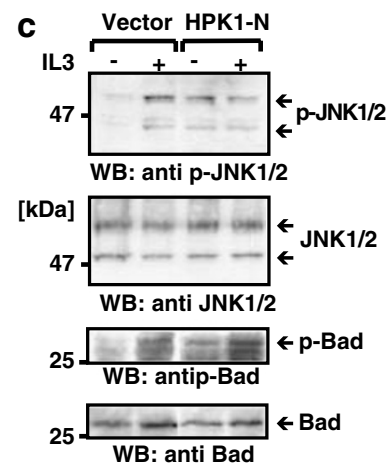

b

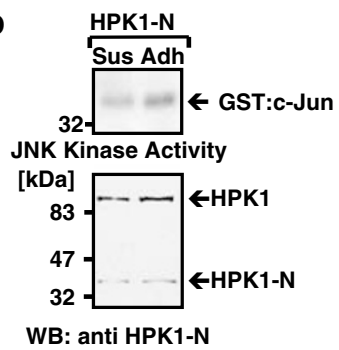

d

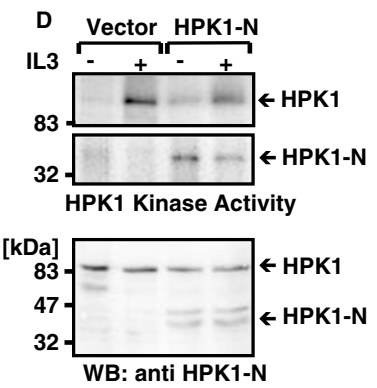

Figure 4 Sustained JNK activation and Bad phosphorylation are mediated by the constitutive, IL-3 independent kinase activity of HPK1-N. (a) HPK1-N, HPK1-C or vector transduced FDC-P1 cell pools were used to precipitate endogenous JNK proteins and determine their kinase activity towards recombinant GST:C-Jun $\mathrm{N}_{\mathrm{N}}$ protein. Phosphorylation of C-Jun $\mathrm{N}_{\mathrm{N}}$ was used to quantify JNK activity relative to vector containing FDC-P1 cells. Expression of endogenous JNK proteins was controlled by Western blotting (WB). (b) Adherent (Adh) HPK1-N cells and HPK1-N cells grown in suspension (Sus) were analyzed as in (a). Expression of HPK1 proteins was controlled by Western blotting. (c) FDC-P1 cell pools harboring HPK1$\mathrm{N}$ or empty vector were cultured without IL-3 for $2 \mathrm{~h}(-)$ or continuously cultured with IL-3 ( + ) and analyzed by Western blotting (WB) using the indicated antibodies. (d) FDC-P1 cell pools as shown in C were deprived of IL-3 for $2 \mathrm{~h}$ and IL-3 was added back for $15 \mathrm{~min}(+)$ or not $(-)$. Endogenous full-length HPK1 (top panel) or ectopically expressed HPK1-N (middle panel) was precipitated and kinase activity was determined by an in vitro kinase assay. Expression of HPK1 proteins in the lysates was controlled by WB

To test whether there is a link between JNK activation and survival of HPK1-N cells without IL-3, we compared the JNK activity of adherent HPK1-N cells and HPK1-N cells growing in suspension. Interestingly, we found a higher constitutive JNK activity in adherent HPK1-N cells (Figure 4b). This correlated with the higher apoptosis-resistance of these cells. This result again points towards a role for sustained JNK activation in cytokine-independent survival of HPK1-N cells.

In hematopoietic progenitor cells, JNK activity is dependent on IL-3 stimulation. ${ }^{23}$ To test, if IL-3 treatment might also induce JNK activity in HPK1-N cells, we deprived the cells from IL-3 and tested phosphorylation of endogenous JNK proteins following readdition of IL-3. While JNK signaling was induced by IL-3 in FDC-P1 cells containing empty vector, HPK1-N cells displayed JNK activity already without IL-3 stimulation (Figure 4c, top panel). In addition, the JNK target $\mathrm{Bad}^{23}$ remained phosphorylated in HPK1-N cells in the absence of IL-3 stimulation (Figure 4c, bottom panel). These results provide a first explanation for the cytokine-independent survival of HPK1-N cells by antiapoptotic phosphoryla-

tion and subsequent sequestration of Bad partly due to sustained JNK activation.

Sustained JNK activation by constitutive kinase activity of HPK1-N. To better understand the role of endogenous HPK1 and HPK1-N as upstream activators of the JNK pathway, ${ }^{7,8}$ we precipitated both HPK1 proteins from IL-3 stimulated or non-stimulated cells and determined their activity by an in vitro kinase assay. As expected, the kinase activity of endogenous full-length HPK1 was found to be IL-3 dependent (Figure 4d, top panel). In contrast, HPK1-N kinase activity was independent of IL-3 and not further enhanced by addition of cytokine (Figure $4 \mathrm{~d}$, middle panel). Our results indicated that in myeloid cells HPK1-N is a constitutive active kinase, while activity of full-length HPK1 is controlled by IL-3. Thereby, deregulated and persistent HPK1-N kinase activity provides an explanation for sustained JNK activation in the absence of IL-3 and a molecular mechanism for myeloid progenitor cell survival during growth factor deprivation and monocytic differentiation.

Blocking of caspase activity reduces monocytic differentiation. To test if caspase activity is required for monocytic differentiation, we applied the pan-specific caspase inhibitor Z-QVD-fmk to our in vitro differentiation system following the 2-day preculture period. On day 4 of differentiation, the addition of QVD had effectively reduced the accumulation of processed caspase-3 (Figure 5a), which was found to be most prominent at this time point before (Figure 1c). Consequently, addition of the caspase inhibitor led to a reduction of HPK1 processing towards HPK1-N (Figure $5 \mathrm{~b}$, top panel), indicating that HPK1 is cleaved by caspases during in vitro differentiation. Furthermore, HPK1$\mathrm{N}$ kinase activity (Figure $5 \mathrm{~b}$, middle panel) and total JNK activity (Figure $5 \mathrm{~b}$, bottom panel) were found to be reduced, indicating that HPK1-N activity contributes to JNK activation in primary mouse progenitor cells during differentiation.

Most strikingly, the presence of the caspase inhibitor zQVD-fmk caused a reduction in the number of differentiating cells in vitro (Figure $5 \mathrm{c}$ ). This reduced differentiation was not due to increased apoptosis as nuclear fragmentation was nearly absent in the presence of $z-Q V D-f m k$ (Figure $5 d$ ). Therefore, HPK1-N kinase activity and sustained JNK activation in the absence of IL-3 are likely regulated by caspase activity and crucial for monocytic differentiation.

\section{Discussion}

We studied myeloid cell differentiation towards the monocytic lineage in vitro to gain a better understanding of the mechanisms of differentiation. We detected a tightly controlled non-apoptotic caspase-3 activity during differentiation of primary mouse progenitor cells resulting in cleavage of HPK1. The N-terminal fragment, HPK1-N, comprising the isolated kinase domain, was shown to mediate robust cytokine-independent JNK activation in FDC-P1 hematopoietic progenitor cells. Consequently, constitutive HPK1-N kinase activity led to sustained JNK activity and induced phosphorylation of Bad. 
a

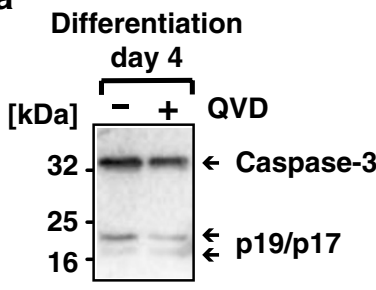

WB: anti Caspase-3

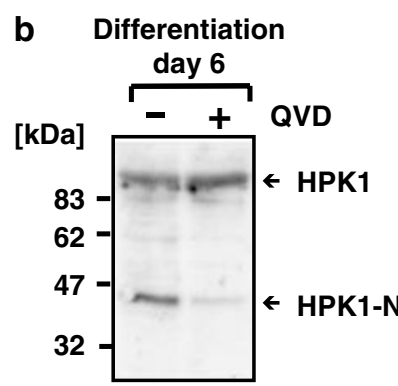

WB: anti HPK1-N

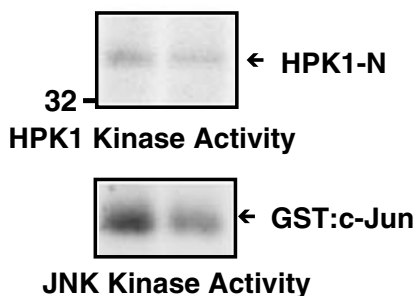

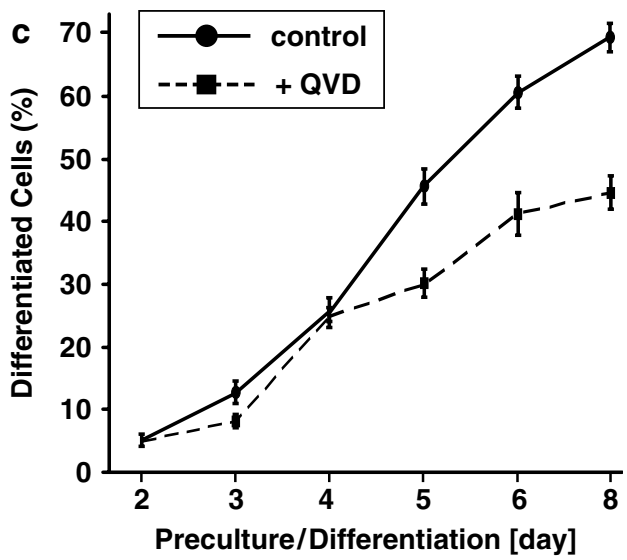

d

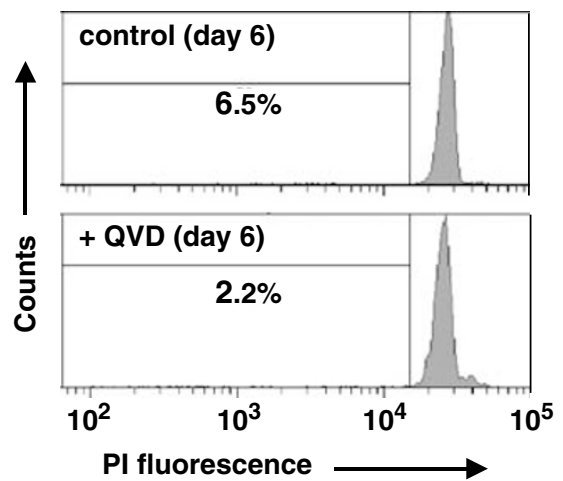

Figure 5 Blockade of caspase activity suppresses HPK1-N cleavage and reduces monocytic differentiation. (a-d) Differentiation of mouse monocytic progenitor cells in vitro was carried out in the presence or absence of the pan-specific caspase inhibitor Z-QVD-fmk. (a and $\mathbf{b}$ ) At the indicated times during in vitro differentiation cell lysates were subjected to Western blotting (WB) using antibodies against caspase-3 or HPK1-N. Endogenous HPK1-N (middle panel) or endogenous JNK proteins (bottom panel) were precipitated and kinase activity was determined by an in vitro kinase assay. Phosphorylation of GST:C-JunN was used to quantify JNK activity. (c) At various times during in vitro differentiation, the percentage of differentiated cells was quantified by the macrophage marker F4/80. Standard deviation was calculated from triplicate analysis. The experiment is representative of three repeats. (d) At day 6 of in vitro differentiation with or without Z-QVD-fmk apoptosis was analyzed by PI-staining. The percentage of cells showing nuclear fragmentation is given

Over the last years evidence for cell-autonomous hematopoietic lineage decisions has accumulated and it appears necessary to prevent apoptosis in order to allow for differentiation of progenitor cells. ${ }^{2}$ Here, we provide an example, how the isolated kinase domain of HPK1, HPK1$\mathrm{N}$, can contribute to monocytic differentiation of hematopoietic progenitor cells by eliciting stimulation-independent JNK activation. This mechanism may contribute to the switch from IL-3 dependent towards IL-3 independent survival during differentiation of primary progenitor cells. Nevertheless, the differentiation of FDC-P1 progenitor cells harboring HPK1-N does not proceed to a final stage of complete IL-3 independent survival (Figure 2d). Furthermore, complementation of HPK1$\mathrm{N}$ cells with the $\mathrm{C}$-terminal HPK1 fragment, HPK1-C, did also not lead to a complete release from cytokine-dependency (data not shown). This clearly indicates that differentiation of progenitor cells does not depend on singular activation of one pathway or protein. In addition to JNK-dependent mechanisms, $\mathrm{PKC} \alpha$ has been shown to suppress apoptosis induced by IL-3 withdrawal ${ }^{26}$ and Akt protein kinase was found to mediate IL-3 dependent survival. ${ }^{27}$ Cell survival during differentiation may involve upregulation of antiapoptotic proteins like $\mathrm{Bcl}-\mathrm{X}_{\mathrm{L}}$ and $\mathrm{XIAP} .{ }^{28}$ While HPK1-N cells already react to $M-C S F$ stimulation (Figure $2 d$ ) and express the $M$ CSFR (Figure 3c), M-CSFR triggered pathways may function inefficiently. $20,29,30$

Caspase cleavage of HPK1 was shown to modulate its function in lymphocytes. ${ }^{8,14}$ In contrast, little is known about the role of HPK1 in myeloid cells. Our report indicates that caspase-mediated cleavage may be a general feature of HPK1 signal transduction. Depending on the cell type, caspase cleavage might uncouple HPK1 activity from antigen-receptor or cytokine-receptor signaling. In lymphocytes, HPK1 cleavage leads to inefficient T-cell receptor stimulation and results in apoptosis-sensitivity. ${ }^{15}$ In myeloid progenitor cells cleaved HPK1 results in constitutive cytokineindependent signaling and enables prolonged cell survival. Similar to our finding, the potential to mediate differentiation of lymphoid and myeloid progenitors has also been shown for constitutively active $\beta$-catenin. ${ }^{31}$

Accumulating evidence suggests that caspases are not exclusively involved in cell death programs, but rather fulfill various important tasks in non-apoptotic processes. While the non-apoptotic functions for caspases might be dependent on 
the cell type they are often linked to developmental programs, such as keratinocyte differentiation. ${ }^{32}$ Commitment to terminal keratinocyte differentiation was reported to be mediated by a caspase-3 dependent inactivation of $\operatorname{PKC} \delta{ }^{33}$ Further examples for non-apoptotic caspase activities include erythrocyte differentiation, ${ }^{34}$ platelet formation ${ }^{35}$ or T-cell activation and proliferation. ${ }^{36,37} \mathrm{~A}$ caspase-like activity was also found to be required for survival of dendritic cells and control of dendritic cell maturation. ${ }^{8,38,39}$ Albeit for myeloid cells the involvement of caspases in monocytic differentiation is most evident, ${ }^{4,5}$ the functional role of the caspase targets recently identified during differentiation of progenitor cell lines is elusive ${ }^{40}$ Therefore, cleavage of full-length HPK1 in primary myeloid progenitor cells and the generation of a constitutively active HPK1-N kinase provide a possible mechanistic explanation for growth factor-independent survival during monocytic differentiation. Functional analyses of additional caspase targets will probably further improve the understanding of monocytic differentiation in the future.

\section{Materials and Methods}

Primary cell culture and generation of stable FDC-P1 cell lines. Total mouse bone marrow or total mouse fetal liver cells were used as starting population for in vitro differentiation. Bone marrow was prepared by syringemediated flushing of marrow-rich bones (e.g. femur, tibia) with cell culture medium. To generate a cell suspension, mouse fetal liver was passed through a nylon mesh (BD Falcon, Cell Strainer $70 \mu \mathrm{M}$ ). Harvested bone marrow or fetal liver cells were washed with cell culture medium and resuspended in ACK buffer $\left(150 \mathrm{mM} \mathrm{NH}_{4} \mathrm{Cl}\right.$, $10 \mathrm{mM} \mathrm{KHCO}_{3}, 1 \mathrm{mM} \mathrm{Na} 2 \mathrm{EDTA}, \mathrm{pH}=7.3$ ) and incubated for $5 \mathrm{~min}$ to lyse erythrocytes. After additional washing, harvested cells were reseeded in cell culture medium and cultivated for 2 days in the presence of IL-3 and M-CSF to enrich for myeloid progenitors. During this preculture period, fibroblast-like cells and predifferentiated monocytic cells adhered to the cell culture dish, while monocytic progenitors remained in suspension. Suspension cells were harvested, IL-3 was removed from the cell culture medium and cells were differentiated for an additional 7 days in cell culture medium supplemented with M-CSF. Primary mouse progenitor cells and the IL-3 dependent mouse hematopoietic progenitor cell line FDC-P1 ${ }^{16}$ were cultured in Dulbecco's modified Eagle's medium (Life Technologies) supplemented with $5 \%$ fetal bovine serum (Sigma), $100 \mu \mathrm{g} / \mathrm{ml}$ penicillin (Life Technologies), $100 \mu \mathrm{g} / \mathrm{ml}$ streptomycin (Life Technologies), $20 \mathrm{mM}$ L-glutamine (Life Technologies), $0.001 \%$ monothioglycerol (Sigma) and 1.5\% conditioned medium of the myeloma cell line X63 Ag8-653 IL-3 as a source of IL-3. ${ }^{17}$ During culture of primary mouse progenitor cells, $10 \%$ conditioned medium of the cell line L929 was added as a source of M-CSF. The caspase inhibitor QVD (Imgenex) was dissolved at $10 \mathrm{mM}$ in DMSO and used at a final concentration of $5 \mu \mathrm{M}$. FDC-P1 cells were infected using transient supernatants of the ecotropic packaging cell line $\mathrm{GP}+\mathrm{E} 86$ transfected with $10 \mu \mathrm{g}$ of empty retroviral vector or vector encoding fulllength HPK1, HPK1-N (aa 1-328) or HPK1-C (aa 271-827), respectively. At $48 \mathrm{~h}$ later, selection with $1 \mathrm{mg} / \mathrm{ml} \mathrm{G} 418$ (Gibco/BRL) was applied for 12 days.

In vitro kinase assays, SAPK activation and western blotting. HPK1 and JNK kinase assays were performed as described before. ${ }^{7}$ Briefly, $10^{7}$ cell were lysed in $1 \mathrm{ml}$ of buffer A $(50 \mathrm{mM}$ Tris/ $\mathrm{HCl}, \mathrm{pH}=8.0,120 \mathrm{mM}$ $\mathrm{NaCl}, 1 \%$ (v/v) NP-40, $200 \mu \mathrm{M} \mathrm{Na}_{3} \mathrm{VO}_{4}, 5 \mathrm{mM}$ DTT, $25 \mathrm{mM} \mathrm{NaF}, 1 \mathrm{mM} \mathrm{PMSF}$ ) and antibodies directed against JNK1/2 (Santa Cruz and Abcam, $10 \mu \mathrm{l}$ each) or HPK1 (rabbit sera no. 5/6, reacting with native full-length HPK1, $20 \mu \mathrm{l}$ ) or HPK1-N (rabbit sera no.9/10, reacting with the native HPK1 N-terminus, $20 \mu l$ ) were used to immunoprecipitate kinase active proteins. After washing with buffer $\mathrm{A}$, the precipitate was incubated in kinase buffer $\left(50 \mathrm{mM}\right.$ Tris/ $\mathrm{HCl}(\mathrm{pH}=8.0), 2 \mathrm{mM} \mathrm{MnCl}_{2}$, $5 \mathrm{mM} \mathrm{MgCl}_{2}, 0.5 \mathrm{mM} \mathrm{DTT}$ ) with $5 \mu \mathrm{Ci}^{32} \mathrm{P}-\gamma$-ATP and GST:C-Jun $\mathrm{N}$ as substrate for $20 \mathrm{~min}$ at $30^{\circ} \mathrm{C}$. Phosphorylated proteins were resolved by SDS-PAGE and exposed to X-ray film (Hyperfilm, Amersham). Polyclonal rabbit sera anti-HPK1 for Western blotting (no. 2 raised against HPK1 N-terminus or no. 7 raised against HPK1 C-terminus) have been described previously. ${ }^{7,8}$ The antibodies used were: anti-caspase-3 (R\&D Systems), anti-JNK (Santa Cruz and Abcam), anti-phospho-
JNK (Cell Signaling Technology), anti-Bad (Santa Cruz), anti-phospho-Bad (Cell Signaling Technology).

Apoptosis, phagocytosis and cell staining. To quantify apoptotic FDC$\mathrm{P} 1$ cells we determined the sub-G1 fraction by flow cytometry. Cells were collected, washed with PBS and fixed by slow application of 4 volumes $70 \%$ (v/v) Ethanol for $30 \mathrm{~min}$ on ice. After washing with PBS fixed cells were incubated with propidium iodide (PI)-staining solution $(20 \mu \mathrm{g} / \mathrm{ml}$ popidium idodide, $200 \mu \mathrm{g} / \mathrm{ml}$ RNase A in PBS) for $30 \mathrm{~min}$ at room temperature. Subsequent analysis was performed using a FACscan (Beckton Dickinson). Apoptotic mouse progenitor cells were detected by Annexin V staining or Pl-staining. ${ }^{18}$ For analysis of phagocytosis, cells were incubated with yellow-green FluoSpheres (Molecular Probes) for $4 \mathrm{~h}$, washed extensively and analyzed by flow cytometry. For May-Grünwald-Giemsa staining FDC-P1 cells were spread on glass slides by cytocentrifugation, air-dried, methanol fixed and stained.

Reverse transcriptase polymerase chain reaction (RT-PCR) analysis. Following reverse transcription PCR reactions of Trizol-purified (Invitrogen) RNA were performed as described. ${ }^{19}$ The following oligonucleotides were used: myeloperoxidase (MPO), cgcttctccttcttcactgg and ctgccattgtcttggaatcg; MCSFR (c-fms), agcttggcccgactctgacacttc and ttggagaggagcacacggttttg; Mac-1 (CD11b), gagcctccctgtcagtgtagtctt and gggggcagcttcattcatc.

Acknowledgements. We are grateful to $\mathrm{H}$ Sauter for expert secretary assistance. This work was supported by grants from the Deutsche Forschungsgemeinschaft (SFB 405, SFB 293), the Wilhelm Sander-Stiftung, the Deutsche Krebshilfe and the European Community.

1. Dexter TM. Growth factors involved in haemopoiesis. J Cell Sci 1987; 88: 1-6.

2. Fairbairn LJ, Cowling GJ, Reipert BM, Dexter TM. Suppression of apoptosis allows differentiation and development of a multipotent hemopoietic cell line in the absence of added growth factors. Cell 1993; 74: 823-832.

3. Rohrschneider LR, Bourette RP, Lioubin MN, Algate PA, Myles GM, Carlberg K. Growth and differentiation signals regulated by the M-CSF receptor. Mol Reprod Dev 1997; 46: $96-103$.

4. Pandey P, Nakazawa A, Ito Y, Datta R, Kharbanda S, Kufe D. Requirement for caspase activation in monocytic differentiation of myeloid leukemia cells. Oncogene 2000; 19 : 3941-3947.

5. Sordet O, Rebe C, Plenchette S, Zermati Y, Hermine O, Vainchenker W et al. Specific involvement of caspases in the differentiation of monocytes into macrophages. Blood 2002; 100: 4446-4453.

6. Dan I, Watanabe NM, Kusumi A. The Ste20 group kinases as regulators of MAP kinase cascades. Trends Cell Biol 2001; 11: 220-230.

7. Kiefer F, Tibbles LA, Anafi M, Janssen A, Zanke BW, Lassam N et al. HPK1, a hematopoietic protein kinase activating the SAPK/JNK pathway. EMBO J 1996; 15 : 7013-7025.

8. Arnold R, Liou J, Drexler HCA, Weiss A, Kiefer F. Caspase-mediated cleavage of hematopoietic progenitor kinase 1 (HPK1) converts an activator of NF kappa B into an inhibitor of NF kappa B. J Biol Chem 2001; 276: 14675-14684.

9. Liou J, Kiefer F, Dang A, Hashimoto A, Cobb MH, Kurosaki T et al. HPK1 is activated by lymphocyte antigen receptors and negatively regulates AP-1. Immunity 2000; 12: 399-408.

10. Liu SK, Smith CA, Arnold R, Kiefer F, McGlade CJ. The adaptor protein Gads (Grb2-related adaptor downstream of Shc) is implicated in coupling hemopoietic progenitor kinase-1 to the activated TCR. J Immunol 2000; 165: 1417-1426.

11. Tsuji S, Okamoto M, Yamada K, Okamoto N, Goitsuka R, Arnold R et al. B cell adaptor containing src homology 2 domain (BASH) links B cell receptor signaling to the activation of hematopoietic progenitor kinase 1. J Exp Med 2001; 194: 529-539.

12. Yu J, Riou C, Davidson D, Minhas R, Robson JD, Julius $M$ et al. Synergistic regulation of immunoreceptor signaling by SLP-76-related adaptor Clnk and serine/threonine protein kinase HPK-1. Mol Cell Biol 2001; 21: 6102-6112.

13. Arnold R, Patzak IM, Neuhaus B, Vancauwenbergh S, Veillette A, Van Lint J et al. Activation of hematopoietic progenitor kinase 1 involves relocation, autophosphorylation, and transphosphorylation by protein kinase D1. Mol Cell Biol 2005; 25: 2364-2383.

14. Schulze-Luehrmann J, Santner-Nanan B, Jha MK, Schimpl A, Avots A, Serfling E. Hematopoietic progenitor kinase 1 supports apoptosis of T lymphocytes. Blood 2002; 100: 954-960.

15. Brenner D, Golks A, Kiefer F, Krammer PH, Arnold R. Activation or suppression of NFkB by HPK1 determines sensitivity to activation-induced cell death. EMBO J 2005; 24: 4279-4290.

16. Dexter TM, Garland J, Scott D, Scolnick E, Metcalf D. Growth of factor-dependent hemopoietic precursor cell lines. J Exp Med 1980; 152: 1036-1047. 
17. Karasuyama $\mathrm{H}$, Melchers $\mathrm{F}$. Establishment of mouse cell lines which constitutively secrete large quantities of interleukin 2, 3, 4 or 5, using modified cDNA expression vectors. Eur $J$ Immunol 1988; 18: 97-104.

18. Nicoletti I, Migliorati G, Pagliacci M, Grignani F, Riccardi C. A rapid and simple method for measuring thymocyte apoptosis by propidium iodide staining and flow cytometry. $J$ Immunol Methods 1991; 139: 271-279.

19. Borrello MA, Phipps RP. Fibroblast-secreted macrophage colony-stimulating factor is responsible for generation of biphenotypic $B$ /macrophage cells from a subset of mouse $B$ lymphocytes. J Immunol 1999; 163: 3605-3611.

20. Bourette RP, Myles GM, Choi JL, Rohrschneider LR. Sequential activation of phoshatidylinositol 3-kinase and phospholipase C-gamma 2 by the M-CSF receptor is necessary for differentiation signaling. EMBO J 1997; 16: 5880-5893.

21. Evans CA, Pierce A, Winter SA, Spooncer E, Heyworth CM, Whetton AD. Activation of granulocyte-macrophage colony-stimulating factor and interleukin-3 receptor subunits in a multipotential hematopoietic progenitor cell line leads to differential effects on development. Blood 1999; 94: 1504-1514

22. Manfredini R, Balestri R, Tagliafico E, Trevisan F, Pizzanelli M, Grande A et al. Antisense inhibition of c-fes proto-oncogene blocks PMA-induced macrophage differentiation in HL60 and in FDC-P1/MAC-11 cells. Blood 1997; 89: 135-145.

23. Yu CF, Minemoto Y, Zhang JY, Liu J, Tang FM, Bui TN et al. JNK suppresses apoptosis via phosphorylation of the proapoptotic Bcl-2 family protein BAD. Mol Cell 2004; 13: 329-340.

24. Wang XN, Studzinski GP. Inhibition of p38MAP kinase potentiates the JNK/SAPK pathwa and AP-1 activity in monocytic but not in macrophage or granulocytic differentiation of HL6O cells. J Cell Biochem 2001; 82: 68-77.

25. Chen YR, Meyer CF, Ahmed B, Yao ZB, Tan TH. Caspase-mediated cleavage and functional changes of hematopoietic progenitor kinase 1 (HPK1). Oncogene 1999; 18 7370-7377.

26. Li WQ, Zhang JC, Flechner L, Hyun T, Yam A, Franke TF et al. Protein kinase C-alpha overexpression stimulates Akt activity and suppresses apoptosis induced by interleukin 3 withdrawal. Oncogene 1999; 18: 6564-6572.

27. Songyang Z, Baltimore D, Cantley LC, Kaplan DR, Franke TF. Interleukin 3-dependent survival by the Akt proteinákinase. Proc Natl Acad Sci 1997; 94: 11345-11350.

28. Miranda MB, Dyer KF, Grandis JR, Johnson DE. The increased lifespan of monocytic versus granulocytic cells is dependent on upregulation of XIAP and $\mathrm{Bcl}-\mathrm{X}-\mathrm{L}$ during monocytic differentiation. Blood 2002; 100: 305A.
29. Bourgin C, Bourette RP, Arnaud S, Liu Y, Rohrschneider LR, Mouchiroud G. Induced Expression and association of the Mona/Gads adapter and Gab3 scaffolding protein during monocyte/macrophage differentiation. Mol Cell Biol 2002; 22: 3744-3756.

30. Liu Y, Jenkins B, Shin JL, Rohrschneider LR. Scaffolding protein Gab2 mediates differentiation signaling downstream of Fms receptor tyrosine kinase. Mol Cell Biol 2001; 21: 3047-3056.

31. Baba Y, Yokota T, Kincade PW. Constitutive signaling via the Wnt/beta-catenin pathway disrupts differentiation and confers multi-lineage differentiation on lymphomyeloid progenitors. FASEB J 2005; 19: A917.

32. Eckhart L, Rendl M, Ban J, Lengauer B, Mayer C, Dockal M et al. Terminal differentiation of human keratinocytes and stratum corneum formation is associated with caspase-14 activation. J Invest Dermatol 2000; 115: 541.

33. Okuyama R, Nguyen BC, Talora C, Ogawa E, di Vignano AT, Lioumi M et al. High commitment of embryonic keratinocytes to terminal differentiation through a notch1caspase 3 regulatory mechanism. Dev Cell 2004; 6: 551-562.

34. Zermati Y, Garrido C, Amsellem S, Fishelson S, Bouscary D, Valensi F et al. Caspase activation is required for terminal erythroid differentiation. J Exp Med 2001; 193: 247-254.

35. de Botton S, Sabri S, Daugas E, Zermati Y, Guidotti JE, Hermine O et al. Platelet formation is the consequence of caspase activation within megakaryocytes. Blood 2002; 100: 1310-1317.

36. Misra RS, Jelley-Gibbs DM, Russell JQ, Huston G, Swain SL, Budd RC. Effector CD4+ T cells generate intermediate caspase activity and cleavage of caspase-8 substrates. $\mathrm{J}$ Immunol 2005; 174: 3999-4009.

37. Su H, Bidere N, Zheng LX, Cubre A, Sakai K, Dale J et al. Requirement for caspase-8 in NF-kappa B activation by antigen receptor. Science 2005; 307: 1465-1468.

38. Franchi L, Condo I, Tomassini B, Nicolo C, Testi R. A caspaselike activity is triggered by LPS and is required for survival of human dendritic cells. Blood 2003; 102: 2910-2915.

39. Santambrogio L, Potolicchio I, Fessler SP, Wong SH, Raposo G, Strominger JL. Involvement of caspase-cleaved and intact adaptor protein 1 complex in endosomal remodeling in maturing dendritic cells. Nat Immunol 2005; 6: 1020-1028.

40. Cathelin S, Rebe C, Haddaoui L, Simioni N, Verdier F, Fontenay M et al. Identification of proteins cleaved downstream of caspase activation in monocytes undergoing macrophage differentiation. J Biol Chem 2006; 281: 17779-17788. 